Bundesgesundheitsbl -

Gesundheitsforsch - Gesundheitsschutz

2000 • 43:407-414 @ Springer-Verlag 2000

Leitthema: Bundes-Gesundheitssurvey

W. Casper

Robert Koch-Institut, Berlin

\title{
Verletzungen und Vergiftungen
}

\section{Ergebnisse aus dem Bundes-Gesundheitssurvey 1998}

\section{Zusammenfassung}

Aus dem Bundes-Gesundheitssurvey 1998 geht hervor, dass $11,9 \%$ der Befragten innerhalb von zwölf Monaten eine Verletzung oder Vergiftung durch Unfall erlitten, die ärztlich versorgt werden musste. Von $0,3 \%$ der Befragten wurde tätlicher Angriff,Schlägerei oder Misshandlung als Ursache von Verletzungen angegeben. Selbsttötungsversuche als Verletzungsursache wurden nicht genannt. Nach einer überschlägigen Hochrechnung der Surveyergebnisse haben in Deutschland im Laufe von zwölf Monaten 8,5 Millionen Personen im Alter von 18 bis 79 Jahren eine Verletzung erlitten. Insgesamt ereigneten sich $28 \%$ aller Unfälle im Haus und in der häuslichen Umgebung, 24\% bei der Arbeit oder auf dem Arbeitsweg, 23\% bei Sport/Spiel, $18 \%$ auf der Straße bzw. dem Gehweg sowie 7\% an sonstigem Unfallort. Männer erleiden Unfallverletzungen häufiger als Frauen bei der Arbeit sowie bei sportlichen Aktivitäten, während Frauen häufiger im Haus und in der häuslichen Umgebung verunfallen. Männer und Frauen in den neuen Bundesländern verzeichnen höhere Anteile bei Arbeitsunfällen und geringere Anteile bei Unfällen durch sportliche Aktivitäten als Männer und Frauen in den alten Bundesländern.Verkehrsunfälle haben in beiden Landesteilen die gleichen Anteile am Unfallgeschehen.

\section{Schlüsselwörter}

Unfallhäufigkeit - Unfallort · Unfallursache · Morbidität · Unfallprävention
V erletzungen und Vergiftungen sind eine häufige Ursache medizinischer Behandlungen, zeitweiliger oder dauernder Arbeitsunfähigkeit und von vorzeitigen Todesfällen. Sie führen zu erheblichen Beeinträchtigungen von Gesundheit und Leistungsfähigkeit der betroffenen Personen und verursachen hohe Kosten im Gesundheitswesen und für die Volkswirtschaft. Mit dem BundesGesundheitssurvey 1998 stehen erstmals bundesweit ärztlich validierte Daten zum Verletzungsgeschehen zur Verfügung, die sowohl einen Überblick über das gesamte Spektrum als auch detaillierte Einblicke ermöglichen.

\section{Definitionen}

Verletzungen und Vergiftungen sind Folgen von unbeabsichtigten (Unfälle) oder beabsichtigten gewalttätigen Einwirkungen auf Personen, wobei jeweils mehrere Verletzungen je Ereignis auftreten können (sog. multitraumatisches Ereignis). Entsprechend den Realitäten war im Fragebogen des Survey 1998 zusätzlich vorgesehen, ggf. mehrere Ereignisse anzugeben (sog. Unfalldichte). Im Ergebnis der Auswertung wurden je Person, die Angaben zu Verletzungen und Vergiftungen gemacht hat, im Durchschnitt 1,1 Ereignisse für die letzten zwölf Monate ermittelt.Aufgrund des vorherrschenden Anteils der Unfallverletzungen beziehen sich die detaillierten Auswertungen hauptsächlich auf die Unfälle. In der Auswertung werden "Ereignis" und "Unfall", "verletzte" und "verunfallte" Person als Synonym gebraucht. Da keine
Studie zu sogenannten "Unfallpersönlichkeiten" auf Grundlage der Surveydaten beabsichtigt ist, ist im Rahmen dieser Auswertung ohne Belang, ob eine Person zwei Unfälle oder zwei Personen je einen Unfall hatten. Relevant für die Auswertung sind die Zahl der Unfälle, die zu Verletzungen und Vergiftungen führen, sowie deren Ursachen und der jeweilige Unfallort.

Die Unfallorte (Unfallkategorien) wurden in Anlehnung an die ICD (EKlassifikation) definiert:

D Haus, häusliche Umgebung,

Derkehr (Straße, Gehweg),

D Arbeitsort/Arbeitsweg,

D Erholungs- und Sportstätte (im Survey zum besseren Verständnis als Sport/Spiel erfragt),

D sonstige (in die anderen Kategorien nicht einzuordnen, z. B. öffentliche Gebäude, offene Gelände, Wälder, Parks, Friedhöfe etc.).

Die Verletzungsarten entsprechen den Diagnosen der Klasse XVII ICD-9, Verletzungen und Vergiftungen.

\section{Material}

In Ermangelung von Daten zum Unfallgeschehen aus bisherigen Surveys werden zum Vergleich und zur Beurteilung von zeitlichen Entwicklungen Daten aus den Mikrozensus zum Gesundheitszustand der Bevölkerung der Jahre 1992

Waldtraut Casper

Robert Koch-Institut, Postfach 650280,

13302 Berlin 
Bundesgesundheitsbl -

Gesundheitsforsch - Gesundheitsschutz

2000 • 43:407-414 @ Springer-Verlag 2000

\section{W. Casper}

\section{Accidents and Poisoning}

Results from the German National Health Interview and Examination Survey 1998

\section{Summary}

From the German National Health Interview and Examination Survey 1998 it emerges that within 12 months $11,9 \%$ of the persons interviewed suffered from an injury or poisoning through accident, which had to be treated medically. $0,3 \%$ of those interviewed reported a violent assault, brawl or maltreatment as cause of their injuries. Suicide-attempts as cause of injuries were not mentioned. By roughly extrapolating the results of the survey to the population at large around 8,5 million persons in the age of 18-79 years are suffering from an injury in Germany per year. Altogether, $28 \%$ of all accidents occurred at home and in domestic surroundings, $25 \%$ were occupational accidents, $23 \%$ occurred during sports activities, $19 \%$ on the streets. Men suffer from accidental injuries more frequently at work as well as during sports activities than women, while women more frequently get injured by accidents at home and in domestic surroundings. Men and women in East Germany report higher proportions of occupational accidents and less accidents through sports activities than men and women in West Germany. Traffic accidents are as frequent in the west as in the east.

\section{Keywords}

Accident-frequency - Place of accident . Cause of accident $\cdot$ Morbidity $\cdot$ Accidentprevention

\section{Leitthema: Bundes-Gesundheitssurvey}

Tabelle 1

Anteil der Verletzten an den befragten Personen des Surveys 1998 in Prozent

Bundesrepublik Deutschland

Neue Alte

Bundesländer

\begin{tabular}{lccccc}
\hline Altersgruppen & insgesamt & männlich & weiblich & insgesamt & insgesamt \\
$<20$ & 24,9 & 33,1 & 16,3 & 23,8 & 27,9 \\
$20-<30$ & 18,7 & 25,5 & 11,7 & 18,7 & 18,9 \\
$30-<40$ & 12,5 & 15,4 & 9,6 & 12,8 & 11,6 \\
$40-<50$ & 10,6 & 13,3 & 7,9 & 11,0 & 9,2 \\
$50-<60$ & 11,2 & 12,0 & 10,4 & 11,3 & 10,8 \\
$60-<70$ & 8,2 & 8,9 & 7,6 & 8,6 & 6,9 \\
$70-<80$ & 8,7 & 8,0 & 9,1 & 8,7 & 8,9 \\
gesamt & 12,2 & 15,0 & 9,6 & 12,3 & 11,7 \\
darunter & 11,9 & 14,6 & 9,3 & 12,1 & 10,9 \\
durch Unfall & & & & &
\end{tabular}

und 1995, aus der Krankenhausstatistik 1997 sowie aus der Todesursachenstatistik 1997 verwendet.

\section{Häufigkeit von Verletzungen und Vergiftungen}

Nach ihren Aussagen haben 11,9\% der befragten Personen innerhalb von zwölf Monaten eine Verletzung oder Vergiftung durch Unfall erlitten, die ärztlich versorgt werden musste. Von $0,3 \%$ der Befragten wurde tätlicher Angriff, Schlägerei oder Misshandlung als Ursache von Verletzungen angegeben. Selbsttötungsversuche als Verletzungsursache wurden nicht genannt. Dies bedeutet, dass $98 \%$ aller angegebenen Verletzungen und Vergiftungen unfallbedingt sind.
Die Unfallhäufigkeiten insgesamt liegen in den alten und neuen Bundesländern auf annähernd gleichem Niveau (Tabelle 1). In den alten Bundesländern haben 15,0\% der Männer und 9,4\% der Frauen Unfallverletzungen erlitten, in den neuen Bundesländern 13,3\% der Männer und 8,7\% der Frauen. Die Verletzungshäufigkeit je verunfallte Person im Zeitraum von zwölf Monaten beträgt im Durchschnitt 1,1.

\section{„Bis zum Alter von 50 Jahren ist die Unfallhäufigkeit der Männer etwa doppelt so hoch wie diejenige der Frauen."}

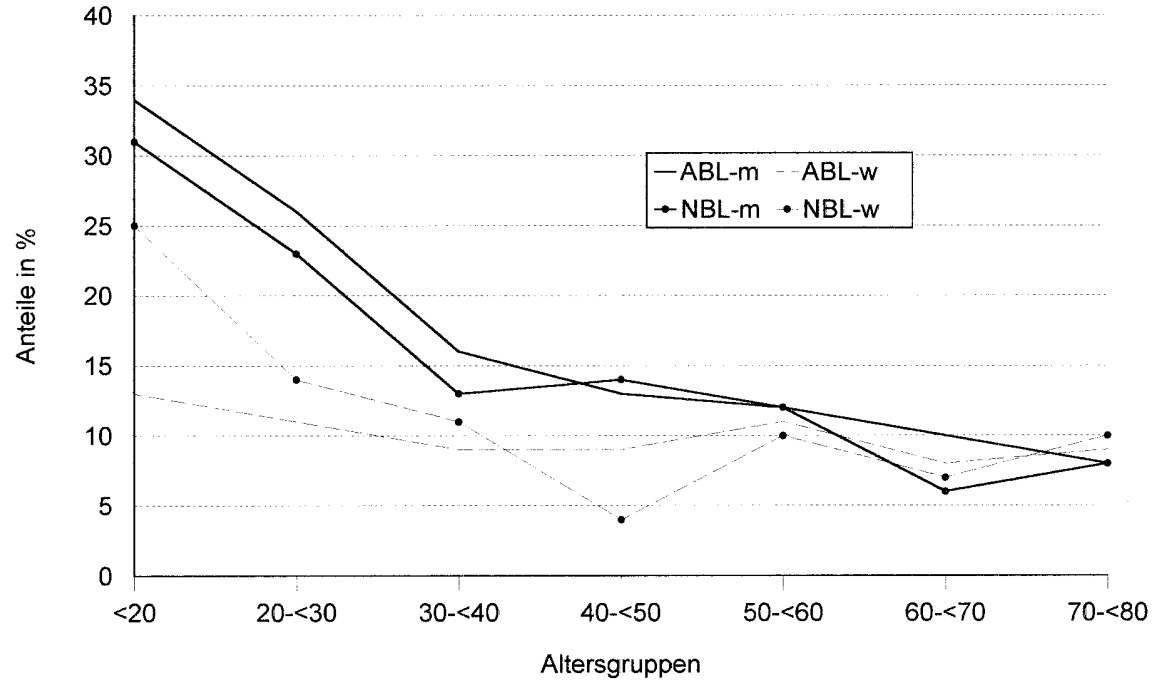

Abb. $1 \Delta$ Anteil der Verletzten an den Befragten nach Geschlecht, alte und neue Bundesländer; Survey 1998 
Betrachtet man die Häufigkeiten der Unfälle nach Alter und Geschlecht, zeigen sich beträchtliche Differenzen (Abb. 1). Gleichermaßen für die alten und die neuen Bundesländer trifft zu:

D Männer verunfallen bis zum Alter von 70 Jahren häufiger als Frauen. Bis zum Alter von 50 Jahren ist die Unfallhäufigkeit der Männer etwa doppelt so hoch wie diejenige der Frauen. Jenseits 70 Jahre überwiegen die Unfallhäufigkeiten der Frauen, jedoch mit deutlich geringeren Unterschieden zu den Männern als vorher umgekehrt.

D Die Unfallhäufigkeiten sind am größten in der jüngsten Altersgruppe. Sie verringern sich mit zunehmendem Alter und steigen erwartungsgemäß in den Altergruppen ab 70 Jahre wieder an.

Im Vergleich zum Mikrozensus 1995 weisen die Ergebnisse des Survey 1998 beim Anteil der Verunfallten an den Befragten sowohl insgesamt als auch für beide Geschlechter geringfügig niedrigere Werte aus. Im Vergleich zu den Ergebnissen des Mikrozensus 1992 weisen die Häufigkeiten 1998 insgesamt sowie bei beiden Geschlechtern einen deutlichen Rückgang auf.

Alters- und geschlechtsspezifisch betrachtet, zeigt sich der Rückgang der Häufigkeiten vom Mikrozensus 1992 zum Survey 1998 vor allem in den mittleren Altersgruppen bei den Männern und in den höheren Altersgruppen bei

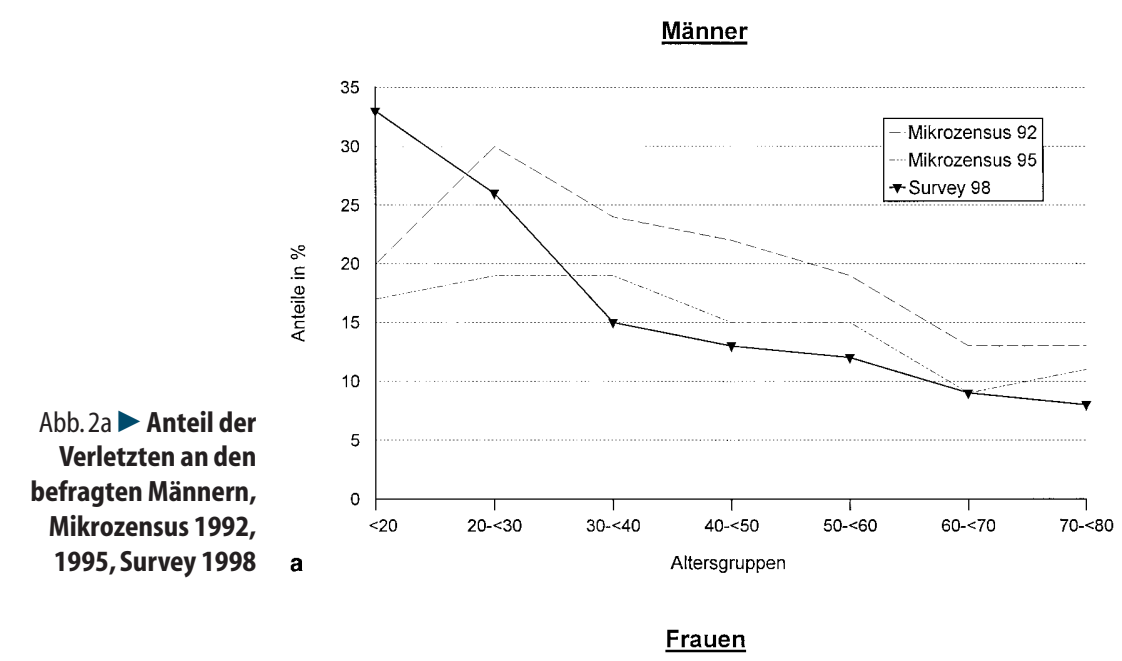

Abb. $2 b>$ Anteil der Verletzten an den befragten Frauen, Mikrozensus 1992, 1995, Survey 1998

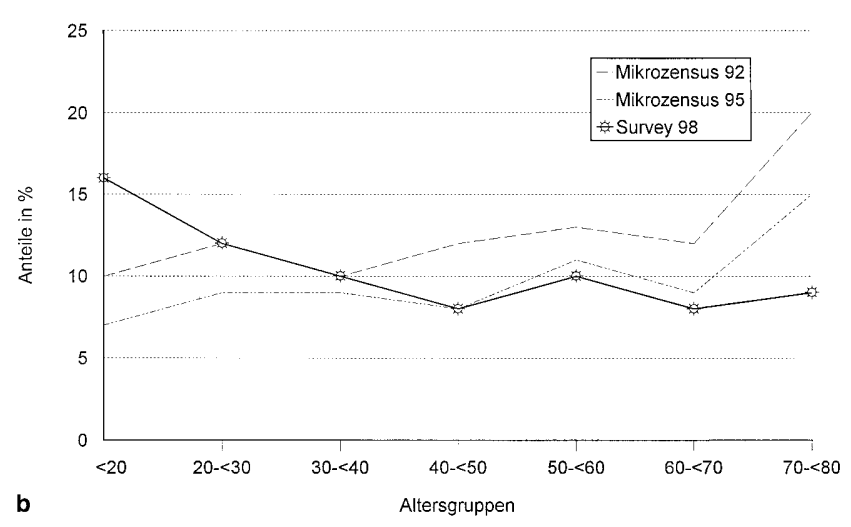

den Frauen. Auffällig sind die besonders großen Differenzen in der jüngsten Altersgruppe sowohl bei den männlichen als auch bei den weiblichen Personen. Hier ist $\mathrm{zu}$ berücksichtigen, dass die jüngste Altersgruppe unterschiedlich

Tabelle 2

Anteil der Verletzten an den befragten Personen 1992, 1995 und 1998 und Entwicklung gegenüber 1992

\begin{tabular}{|c|c|c|c|c|c|}
\hline \multirow[b]{2}{*}{ Altersgruppen } & \multirow{2}{*}{$\begin{array}{l}\text { Mikrozensus } \\
1992 \\
\%\end{array}$} & \multirow{2}{*}{$\begin{array}{l}\text { Mikrozensus } \\
1995 \\
\%\end{array}$} & \multicolumn{3}{|c|}{ Survey 1998} \\
\hline & & & $\begin{array}{l}\text { Index } \\
1992=100\end{array}$ & $\%$ & $\begin{array}{l}\text { Index } \\
1992=100\end{array}$ \\
\hline$<20$ & 15,0 & 12,3 & 82 & 24,9 & 166 \\
\hline $20-<30$ & 20,9 & 14,6 & 70 & 18,7 & 89 \\
\hline $30-<40$ & 17,1 & 14,1 & 82 & 12,5 & 73 \\
\hline $40-<50$ & 17,3 & 11,5 & 66 & 10,6 & 61 \\
\hline $50-<60$ & 16,1 & 12,8 & 80 & 11,2 & 70 \\
\hline $60-<70$ & 12,6 & 8,7 & 69 & 8,2 & 65 \\
\hline $70-<80$ & 17,7 & 13,3 & 75 & 8,7 & 49 \\
\hline gesamt & 17,0 & 12,6 & & & \\
\hline $\begin{array}{l}\text { standardisiert auf } \\
\text { die Altersgruppen- } \\
\text { struktur der Befrag- } \\
\text { ten des Survey } 1998\end{array}$ & 16,9 & 12,5 & 74 & 12,2 & 72 \\
\hline
\end{tabular}

strukturiert ist (im Survey 18 bis $<20$ Jahre und im Mikrozensus 15 bis $<20$ Jahre). Es gibt Anhaltspunkte, dass bei gleichstrukturierten Altersgruppen auch die Werte nach dem Mikrozensus höher ausfallen würden. Der geplante Kinder- und Jugend-Gesundheitssurvey wird detaillierte Aussagen dazu ermöglichen (Tabelle 2, Abb. 2a, 2b).

\section{Unfallort}

Nach dem Survey 1998 ereigneten sich insgesamt $28 \%$ aller Unfälle im Haus und in der häuslichen Umgebung, $24 \%$ der Unfälle bei der Arbeit oder auf dem Arbeitsweg, 23\% Unfälle bei Sport/Spiel, $18 \%$ auf der Straße bzw. dem Gehweg und $7 \%$ an sonstigem Unfallort. Damit verzeichnen die Surveyergebnisse im Vergleich zu den Ergebnissen der Mikrozensus 1992 und 1995 bei den Arbeits- und Wegeunfällen sowie an sonstigem Ort einen anteilmäßigen Rückgang. Bei den häuslichen Unfällen dagegen ist ein bedeutender Anstieg zu verzeichnen, verursacht durch einen deutlich höheren Anteil von Hausunfällen bei den Männern. Eine Erklärung dafür 
könnte die in diesem Zeitraum um 15\% gestiegene Anzahl von Männern im Alter $\mathrm{ab} 60$ Jahre sein.

\section{„Männer verunfallen häufiger als Frauen bei der Arbeit und bei sportlichen Aktivitäten, Frauen häufiger im Haus und in der häuslichen Umgebung."}

Männer erleiden Unfallverletzungen häufiger als Frauen bei der Arbeit sowie bei sportlichen Aktivitäten, während Frauen häufiger im Haus und in der häuslichen Umgebung verunfallen. Insoweit bestätigen die Daten des Survey zum einen, dass Männer oft risikoreichere Arbeitstätigkeiten verrichten als Frauen und zum anderen die hierzulande übliche Arbeitsteilung außerhalb des Berufslebens zwischen den Geschlechtern (Abb. 3).

Im Vergleich der prozentualen Aufgliederung der Unfälle nach dem Ereignisort zeigen sich zudem deutliche Unterschiede zwischen den alten und neuen Bundesländern (West und Ost). Männer und Frauen im Osten haben mehr Arbeitsunfälle und weniger Unfälle durch sportliche Aktivitäten. Verkehrsunfälle haben in West und Ost die gleichen Anteile am Unfallgeschehen, sind aber im Osten verglichen mit einer repräsentativen Morbiditätsstudie [1] im Kreis Zittau (Land Sachsen) aus 1987/88 um rund ein Drittel angestiegen. Im Vergleich zur Zittauer Studie ergibt sich des weiteren ein Rückgang des Anteils von

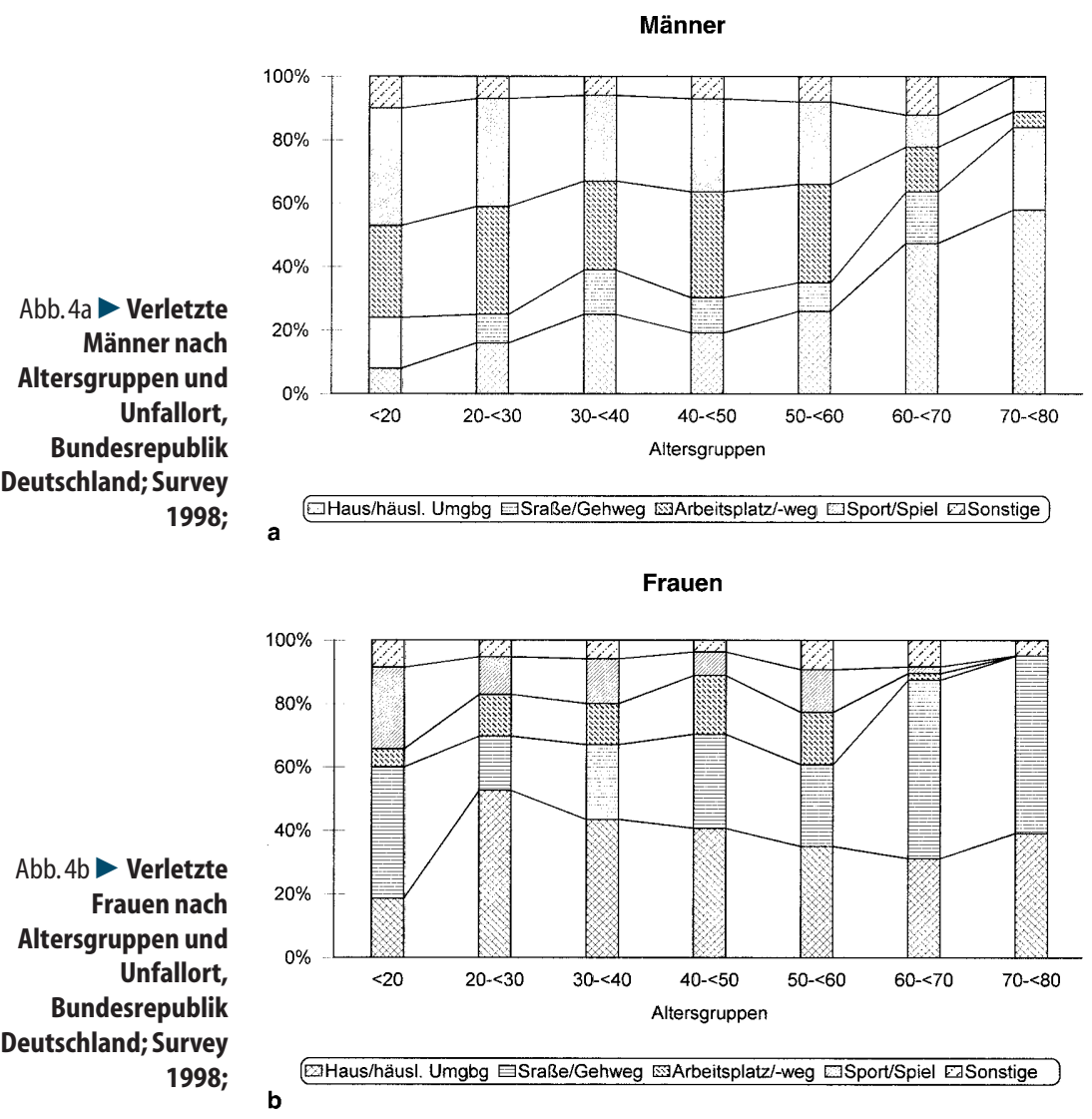

Unfällen im Haus und in häuslicher Umgebung um etwa ein Viertel, was auf die generell bessere Ausstattung mit technischen Geräten und deren vergleichsweise höheren Sicherheitsstandards zurückzuführen sein dürfte. Demgegenüber ist der Anteil von Unfällen bei sportlichen Betätigungen deutlich gestiegen, was auf eine Veränderung des Lebensstils bezüglich sportlicher Aktivitäten in den neuen Bundes-

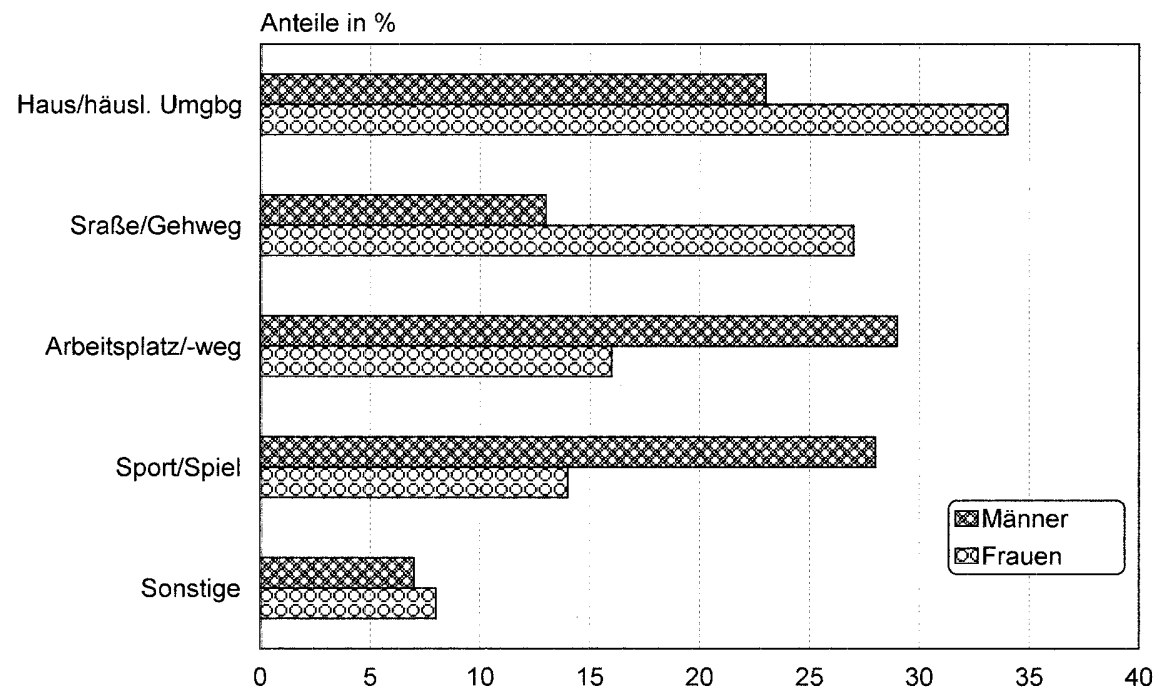

Abb. $3 \Delta$ Verletzte nach Ereignisort und Geschlecht, Bundesrepublik Deutschland; Survey 1998 ländern in den letzten zehn Jahren hindeutet.

Männer im jüngeren Alter, bis etwa 30 Jahre, verzeichnen den höchsten Anteil an Unfällen mit mehr als einem Drittel bei sportlichen Aktivitäten gefolgt von Arbeitsunfällen. In den mittleren Altersgruppen, bis etwa 50 Jahre, ist der Anteil der Arbeitsunfälle am größten. Im Alter ab 60 Jahren dominieren die Hausunfälle mit bis zu zwei Dritteln in der höchsten Altersgruppe. Frauen in der jüngsten Altersgruppe verzeichnen den höchsten Anteil an Unfällen mit einem Drittel ebenfalls beim Sport. In den Altersgruppen zwischen 20 und 60 Jahren stehen Hausunfälle an erster Stelle. Im höheren Alter ab 60 Jahren erleiden die Frauen mehr als die Hälfte der Unfälle im Verkehr, mit steigendem Alter häufiger auf dem Gehweg als auf der Straße (Abb. 4a, 4b).

\section{Verletzungen durch Sturz}

Stürze (einbezogen Stolpern, Ausgleiten, Hinfallen) verursachen rund zwei Drittel aller Unfallverletzungen. Anders als bei Todesfällen infolge Sturz sind nach dem Survey Verletzungen durch Sturz in allen Altersgruppen und bei 


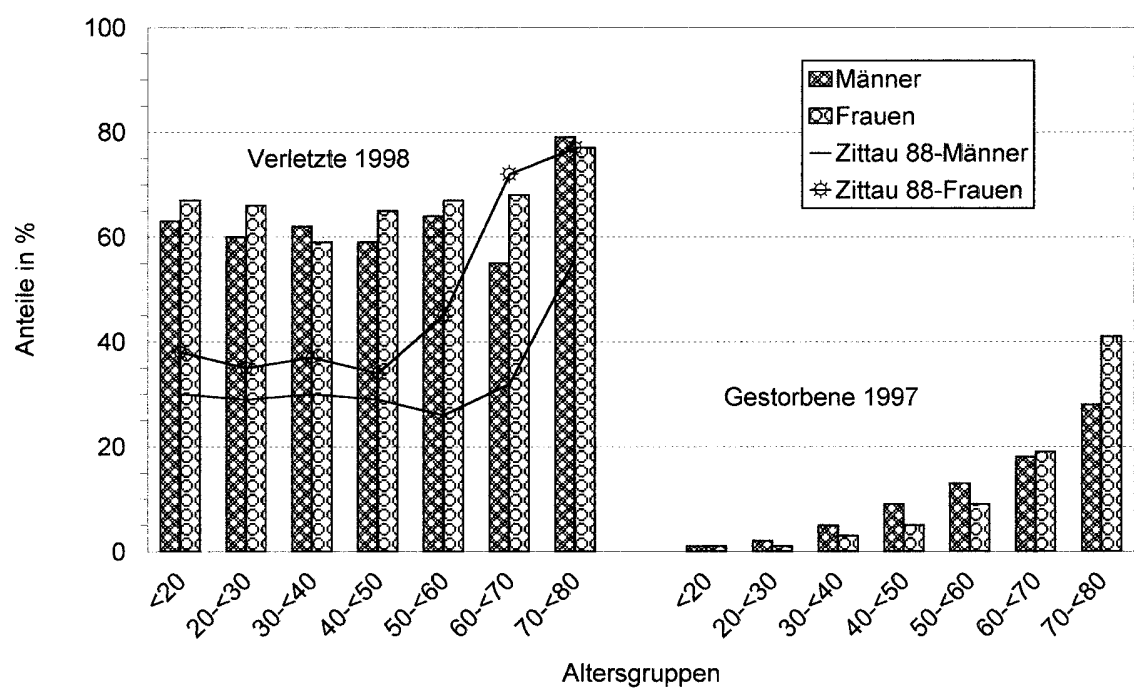

Abb. 5 Anteil der Unfallursache Sturz an allen Unfällen nach Altersgruppen und Geschlecht, Verletzte: Survey 1998, Gestorbene: Todesursachenstatistik 1997, Bundesrepublik Deutschland

beiden Geschlechtern mit annähernd gleichen Anteilen vertreten. Es ist zu vermuten, dass die im Vergleich zur Zittauer Studie höheren Anteile von Stürzen an den Unfällen der mittleren Altersgruppen mit allgemein häufigeren sportlichen Aktivitäten erklärt werden können. Die höchsten Anteile der zu Verletzungen und Todesfällen führenden Stürze verzeichnen erwartungsgemäß die über 70-Jährigen (Abb. 5).

\section{„Stürze verursachen in allen Altersgruppen und bei beiden Geschlechtern zu annähernd gleichen Anteilen rund zwei Drittel aller Unfallverletzungen."}

In den alten Bundesländern ereignen sich Stürze männlicher Personen am häufigsten bei sportlichen Aktivitäten und in den neuen Bundesländern am Arbeitspatz/auf dem Arbeitsweg. Stürze bei den Frauen in West und Ost ereignen sich am häufigsten im Haus und in häuslicher Umgebung, gefolgt von Stürzen auf der Straße bzw. dem Gehweg. Im Alter von 60 Jahren und mehr ziehen sich sowohl Männer als auch Frauen in West und Ost Sturzverletzungen größtenteils in Haus und häuslicher Umgebung zu. Bedeutsam für das Gesundheitswesen ist, dass nach den Ergebnissen des Survey 1998 in den erfassten Altersgruppen jährlich rd. 10\% der männlichen und $7 \%$ der weiblichen
Bevölkerung einen Sturzunfall erleiden. Vier Fünftel aller Frakturen, drei Viertel der Gehirnerschütterungen und zwei Drittel sämtlicher Verstauchungen/Zerrungen/oberflächlichen Verletzungen sind lt. Survey auf Sturzunfälle zurückzuführen.

\section{Verletzungen durch tätlichen Angriff, Schlägerei oder Misshandlung}

Tätlicher Angriff, Schlägerei oder Misshandlung wurde von $0,3 \%$ der Befragten ( $0,4 \%$ der Männer und 0,3\% der Frauen) als Verletzungsursache angegeben. Den höchsten Anteil verzeichnen die unter 20-jährigen männlichen Personen mit 3,0\% und die gleichaltrige Gruppe der weiblichen Personen mit 2,1\%. Ab dem Alter von 60 Jahren wurden diese Ursachen im Survey nicht mehr genannt.

\section{Diagnosen der Unfallverletzungen und Vergiftungen (Verletzungsarten)}

Nach den Ergebnissen des Survey wurden im Durchschnitt bei Männern 1,28 und bei Frauen 1,21 Verletzungen je Unfall ermittelt. Als häufigste Verletzungsfolgen wurden die vergleichsweise weniger schweren Verletzungsarten (Verstauchungen, Zerrungen, offene Wunden, oberflächliche Verletzungen, Quetschungen) angegeben. Auf diese Diagnosengruppen entfallen bei Männern
$66 \%$ und bei Frauen $60 \%$ der Verletzungen. Nächsthäufige Verletzungsart sind Frakturen, von denen nach dem Survey 1998 hochgerechnet 3,6\% der männlichen und 2,8\% der weiblichen Bevölkerung betroffen sind. Ab dem Alter von 70 Jahren bei den Männern und 50 Jahren bei den Frauen stehen die Frakturen anteilmäßig an der Spitze der Verletzungsarten. Die häufigsten Frakturen sind die der unteren Gliedmaßen. Schenkelhalsfrakturen gaben $0,8 \%$ der Männer und 1,2\% der Frauen an. Eine Gehirnerschütterung erlitten Personen beiderlei Geschlechts mit jeweils 6\% der Verletzungsfolgen. Die Gehirnerschütterung ist unter den Unfallfolgen der häufigste Grund für eine Krankenhausbehandlung. Sie nimmt unter allen Behandlungsdiagnosen der Krankenhauspatienten den 6. Rangplatz der Häufigkeit ein.

\section{„Die Gehirnerschütterung ist unter den Unfallfolgen der häufigste Grund für eine Krankenhausbehandlung."}

Verbrennungen oder Verätzungen als Unfallfolge nannten $3 \%$ der im Survey befragten Männer und $4 \%$ der Frauen: Verbrennungen traten am häufigsten in den jüngeren Altersgruppen auf. Dies stimmt überein mit den Ergebnissen der Zittauer Studie. Vergiftungen gaben 2\% der Männer und 4\% der Frauen als Unfallfolge an, und zwar überwiegend Männer und Frauen in den mittleren Altersgruppen. Auch das entspricht den Zittauer Ergebnissen (Tabelle 3).

\section{Arbeitsunfähigkeit}

Die Darstellung verletzungsbedingter Verluste erfolgt an Hand der Arbeitsunfähigkeitstage für die Altersgruppen 20 bis 60 Jahre. Höchste Anteile verzeichnen in West wie Ost die Männer der Altersgruppen 30 bis 50 Jahre und die Frauen im Alter von 50 bis 60 Jahren mit jeweils etwa einem Viertel aller Ausfalltage. Die durchschnittliche Arbeitsunfähigkeitsdauer für Männer ist mit $27 \mathrm{Ta}-$ gen in West und 28 Tagen in Ost annähernd gleich. Bei den Frauen dagegen differieren die Werte deutlich: 23 Tage in West und 27 Tage in Ost. Mit Ausnahme 


\section{Leitthema: Bundes-Gesundheitssurvey}

Tabelle 3

Ausgewählte Verletzungsarten in Prozent der Verletzungen gesamt je Altersgruppe und Geschlecht

\begin{tabular}{|c|c|c|c|c|c|c|c|c|}
\hline Altersgruppen & Frakturen & $\begin{array}{c}\text { darunter } \\
\text { Fraktur der } \\
\text { oberen } \\
\text { Gliedmaßen }\end{array}$ & $\begin{array}{l}\text { Schenkelhals- } \\
\text { fraktur }\end{array}$ & $\begin{array}{l}\text { Fraktur der } \\
\text { unteren } \\
\text { Gliedmaßen }\end{array}$ & $\begin{array}{l}\text { Gehirn- } \\
\text { erschütterung }\end{array}$ & $\begin{array}{c}\text { Verstauchung } \\
\text { Zerrung }\end{array}$ & $\begin{array}{c}\text { offene Wunde } \\
\text { oberflächliche } \\
\text { Verletzung } \\
\text { Quetschwunde }\end{array}$ & $\begin{array}{c}\text { sonstige } \\
\text { Verlet- } \\
\text { zungen }\end{array}$ \\
\hline & & & & $\%$ & & & & \\
\hline \multicolumn{9}{|l|}{ Männer } \\
\hline$<20$ & 7 & 4 & 0 & 4 & 4 & 52 & 23 & 14 \\
\hline $20-<30$ & 11 & 3 & 1 & 4 & 5 & 45 & 32 & 7 \\
\hline $30-<40$ & 22 & 2 & 1 & 12 & 5 & 43 & 26 & 4 \\
\hline $40-<50$ & 23 & 4 & 1 & 6 & 7 & 34 & 20 & 16 \\
\hline $50-<60$ & 27 & 7 & 0 & 11 & 2 & 33 & 33 & 5 \\
\hline $60-<70$ & 21 & 9 & 2 & 9 & 9 & 23 & 34 & 13 \\
\hline $70-<80$ & 39 & 18 & 4 & 11 & 14 & 18 & 18 & 11 \\
\hline gesamt & 20 & 5 & 1 & 8 & 6 & 38 & 28 & 8 \\
\hline \multicolumn{9}{|l|}{ Frauen } \\
\hline$<20$ & 9 & 6 & 0 & 0 & 12 & 42 & 24 & 13 \\
\hline $20-<30$ & 16 & 1 & 1 & 10 & 3 & 41 & 30 & 10 \\
\hline $30-<40$ & 18 & 5 & 1 & 6 & 0 & 46 & 22 & 14 \\
\hline $40-<50$ & 22 & 2 & 0 & 13 & 7 & 31 & 27 & 13 \\
\hline $50-<60$ & 26 & 10 & 2 & 15 & 7 & 39 & 18 & 10 \\
\hline $60-<70$ & 44 & 19 & 2 & 16 & 5 & 30 & 14 & 7 \\
\hline $70-<80$ & 38 & 23 & 0 & 13 & 11 & 23 & 23 & 5 \\
\hline gesamt & 24 & 8 & 1 & 11 & 6 & 37 & 23 & 10 \\
\hline
\end{tabular}

der 50- bis 60-jährigen westdeutschen Frauen (56 Tage) und der 20- bis 30-jährigen ostdeutschen Frauen (36 Tage) übersteigt die Arbeitsunfähigkeitsdauer der Männer in allen Altersgruppen diejenige der Frauen.
Die zu Arbeitsausfällen führenden Verletzungen wurden im Survey auch nach Ereignisorten erfasst. Bemerkenswert ist, dass bei den Männern über $60 \%$ der Ausfalltage infolge von Unfällen auf Arbeits- und Sportunfälle zu-

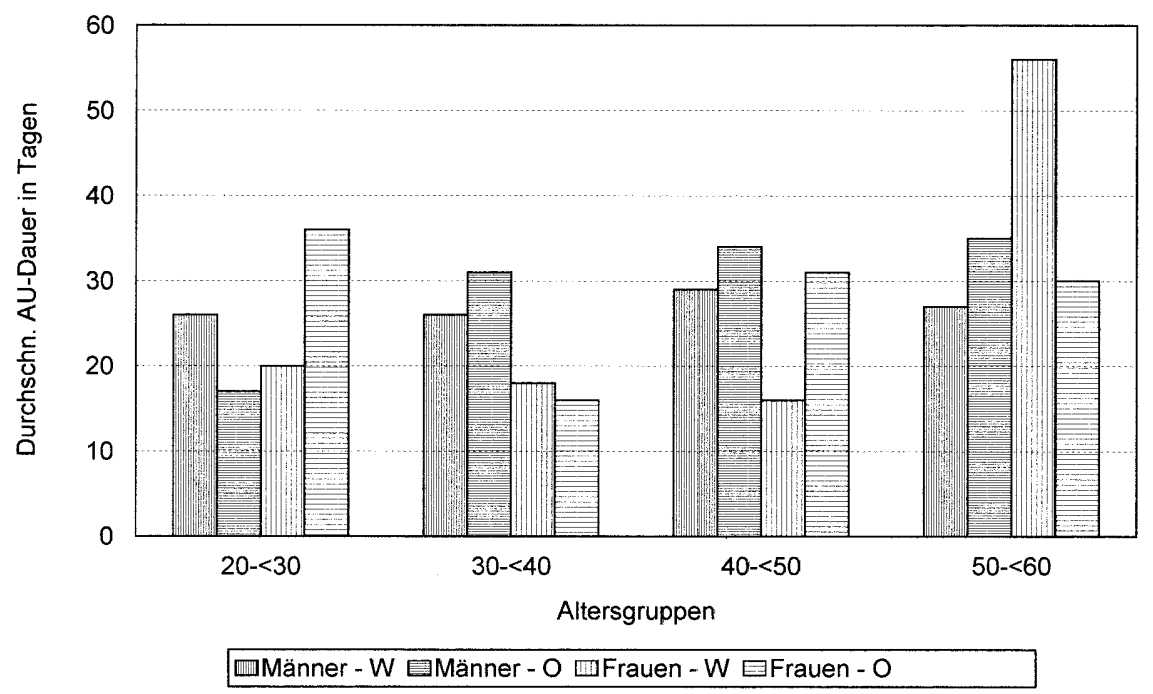

Abb. 6 Durchschnittliche Arbeitsunfähigkeitsdauer in Tagen nach Altersgruppen und Geschlecht, alte Bundesländer, neue Bundesländer; Survey 1998 rückzuführen sind. Bei den Frauen sind die höchsten Anteile an Ausfalltagen mit zusammen $57 \%$ durch Hausunfälle und Unfälle auf der Straße/dem Gehweg verursacht. Die Unterschiede zwischen den alten und neuen Bundesländern sind aus der nachfolgenden Abbildung ersichtlich (Abb. 6).

\section{Hochrechnung}

Aus der Hochrechnung der Surveyergebnisse ergibt sich, dass in Deutschland innerhalb eines Jahres 8,3 Millionen Personen im Alter von 18 bis 79 Jahren eine Unfallverletzung erleiden, davon 5,1 Millionen Männer und 3,2 Millionen Frauen. Das bedeutet, dass die Unfallhäufigkeit in der Bevölkerung höher ist als nach den Schätzungen (8,9 Millionen für alle Altersgruppen) im Gesundheitsbericht für Deutschland bisher angenommen wurde. Insbesondere die Zahl von Haus- und Freizeitunfällen ist lt. Survey höher anzusetzen. Der Vergleich mit dem Gesundheitsbericht zeigt nur für 


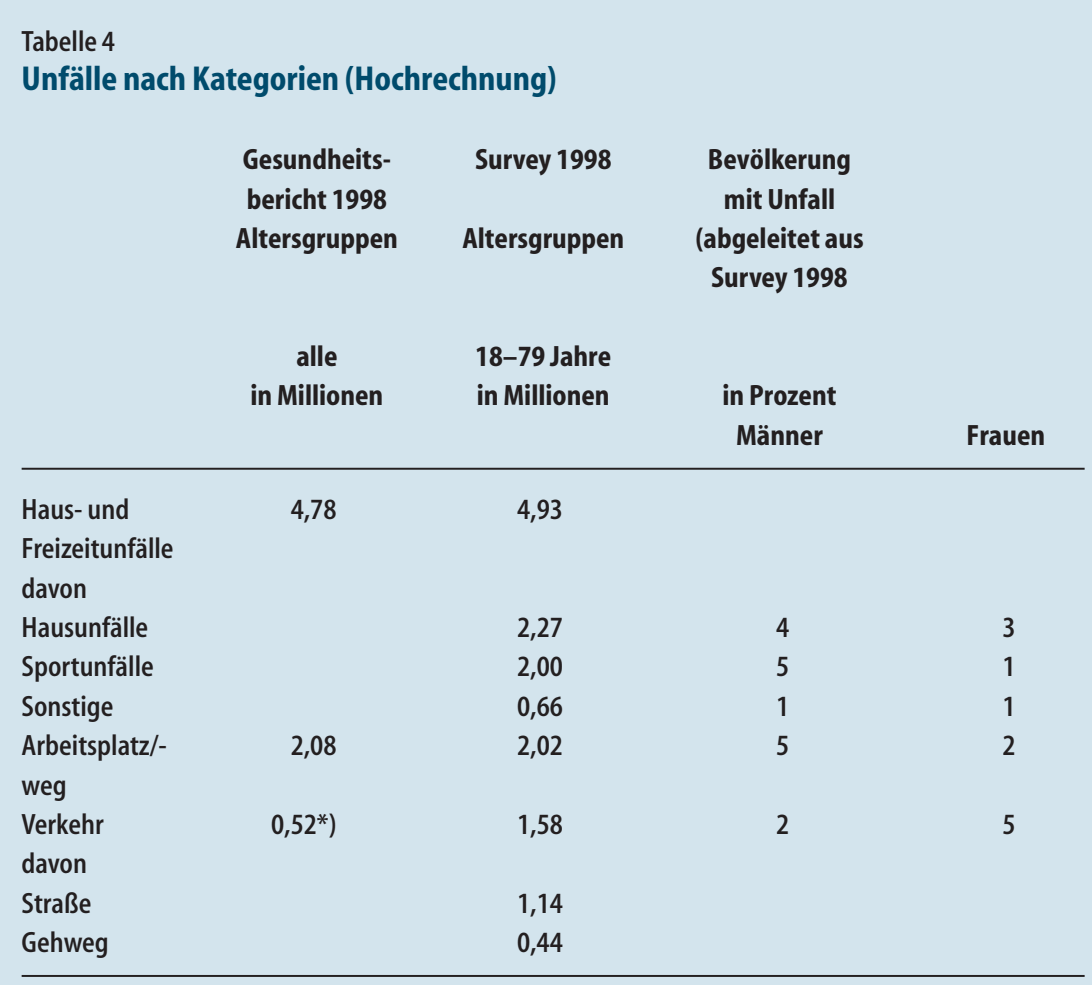

*) Nur polizeilich erfasste Verkehrsunfälle mit Personenschaden

Arbeitsunfälle etwa gleiche Ergebnisse (Tabelle 4).

\section{„Die Zahl aller auf Straße und Gehweg Verletzten macht nach den Ergebnissen des Gesundheitssurveys das Dreifache der in der Polizeistatistik erfassten Fälle aus."}

Die Abweichung bei Verkehrsunfällen fällt dramatisch hoch aus. Im Gesundheitsbericht sind offensichtlich nur die Verletzten berücksichtigt, die bei polizeilich erfassten Verkehrsunfällen registriert wurden. Viele auf der Straße verletzte Fußgänger und Radfahrer werden bekanntermaßen polizeilich nicht erfasst. Aus der vorstehenden Gegenüberstellung ist zu ersehen, dass die Zahl aller auf Straße und Gehweg Verletzten das Dreifache der in der Polizeistatistik erfassten ausmacht. In künftigen Gesundheitsberichten ist dieser Sachverhalt vor allem wegen der Einschätzung der im Gesundheitswesen vorzuhaltenden Kapazitäten und unter präventiven Aspekten zu berücksichtigen. Die Daten Todesursachenstatistik 1997) sachenstatistik. Darüber hinaus enthalten lediglich die Statistiken über Arbeitsunfälle und polizeilich erfasste Verkehrsunfälle einige Aussagen zu Verletzten. Erst die Ergebnisse des Surveys geben einen Überblick über das gesamte Spektrum des nichttödlichen Unfallgeschehens und ermöglichen detaillierte Aussagen. Der Vergleich der Verletzten und Gestorbenen am Unfallort lässt die Schwere der jeweiligen Unfälle deutlich werden (Abb. 7). Die Surveyergebnisse setzen hinsichtlich Prävention neue Schwerpunkte, indem sie aufzeigen, dass auch die bisher weniger beachteten und anzahlmäßig unterschätzten Unfälle ein großes Gewicht im Unfallgeschehen haben und bedeutende Kapazitäten in der medizinischen Betreuung binden. So verursachen $\mathrm{z}$. B. Sportunfälle $2 \% \mathrm{der}$ unfallbedingten Sterbefälle aber $27 \%$ der Verletzungen (letztere bisher statistisch nicht erfasst); Unfälle in Haus und Umgebung $17 \%$ der unfallbedingten Sterbefälle aber $27 \%$ der Verletzungen (letztere ebenfalls bisher statistisch nicht erfasst).

\section{Prävention}

Rund $60 \%$ der Unfallverletzungen und 50\% der Todesfälle durch Unfall sind die Folgen von Haus- und Freizeitunfällen (Unfälle in Haus und häuslicher Umgebung, bei Sport und an sonstigen Orten). Das ist ein bedeutender Anteil, der größerer Aufmerksamkeit bedarf. Insbesondere die Hausunfälle betreffen ältere Personen, die oft bereits durch Krank-

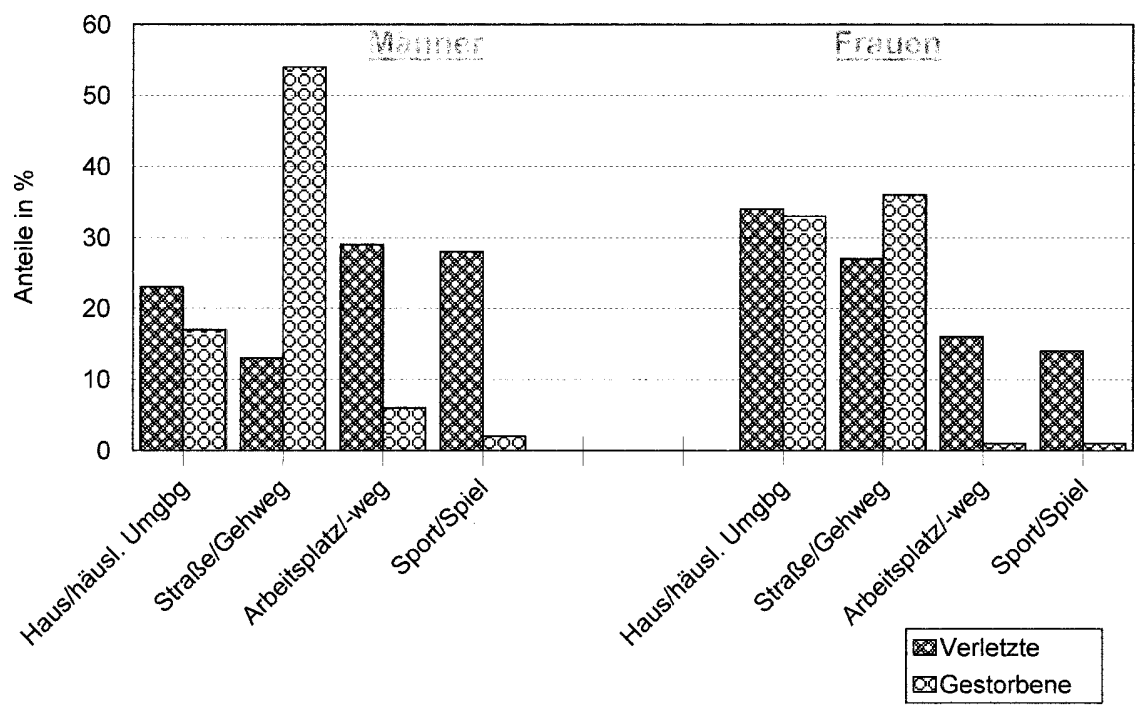

Abb. 7 Verletzte und Gestorbene nach Geschlecht und Unfallort (Survey 1998 , 
Buchbesprechung

heiten physisch und psychisch beeinträchtigt und deshalb erhöhten Unfallrisiken ausgesetzt sind [2]. Für die Analyse von Verkehrs- und Arbeitsunfällen existieren Institutionen, deren Aufgabe sich auch auf Prävention erstreckt.

\section{„Die Möglichkeiten für eine bessere Prävention von Hausunfällen werden in Deutschland noch nicht voll ausgeschöpft."}

Für eine bessere Prävention vor allem von Hausunfällen gibt es gute Möglichkeiten, die in einigen EU-Ländern beispielhaft genutzt, in Deutschland jedoch nicht ausgeschöpft werden (z. B. "Sichere Gemeinden" in Österreich [3], "Safe Community" in Schweden [4]). Technische Sicherheitsstandards allein reichen nicht aus. Für die Prävention von Unfällen vor allem älterer Personen ist auch unter Berücksichtigung des steigenden Anteils dieser Personengruppe an der Bevölkerung mehr gesellschaftliches Engagement erforderlich. Unfallprävention sollte als eine Aufgabe der gesamten Gesellschaft angenommen und organisiert werden. Die Erfolge von Programmen zur Unfallprävention in Ländern wie Schweden, Norwegen, Niederlande, Österreich resultieren nicht zuletzt aus gesellschaftlich getragenen Initiativen und verantwortungsvollem Handeln der gesamten Öffentlichkeit.

\section{Literatur}

1. Casper W,Verletzungen und Vergiftungen. In: Das Gesundheitswesen 1990. Institut für Medizinische Statistik und Datenverarbeitung, Berlin: Eigenverlag

2. Casper W (1994) Unfallbedingte Mortalität und Morbidität im höheren Lebensalter. RKI-Tätigkeitsbericht 1994

3. Institut Sicher Leben (1998) INFO/Nr.31, Wien: Eigenverlag

4. Karolinska Institutet, Department of Social Medicine, Kronan Health Centre (1992) Second International Safe Communities Conference September 7-9. KI Red Report 280, Sundyberg 1993.

\section{Weiterführende Literatur}

Ministerium für Gesundheitswesen der DDR: Internationale Klassifikation der Krankheiten, Verletzungen und Todesursachen (IKK) der Weltgesundheitsorganisation (WHO), 9. Revision. Berlin 1981

Niehoff, J.-U.:Sozialmedizin systematisch. UNI-MED Verlag AG, 1995

\section{Quellen}

Statistisches Bundesamt: Fragen zur Gesundheit, Fachserie 12, Reihe S.3, 1992

Statistisches Bundesamt: Fragen zur Gesundheit, Fachserie 12, Reihe S.3, 1995

Statistisches Bundesamt:Todesursachen in Deutschland, Fachserie 12, Reihe 4, 1997

Statistisches Bundesamt: Diagnosedaten der Krankenhauspatienten, Fachserie 12, Reihe 6.2,1997

Statistisches Bundesamt: Gesundheitsbericht für Deutschland, 1998

Statistisches Bundesamt: Statistisches Jahrbuch 1994

Statistisches Bundesamt: Statistisches Jahrbuch 1999
Hrsg.: Th. Eikmann, U. Heinrich, B. Heinzow,

R. Konitzka

Gefährdungsabschätzung von Umweltschadstoffen (GvU).

Ergänzbares Handbuch toxikologischer Basisdaten und ihre Bewertung. (Loseblattsammlung i. Spezialordner, einschl. der 1. Ergänzungslieferung) Erich Schmidt, Berlin Bielefeld München: 790 S., (ISBN 3-503-05083-3), DM 178,-

Zur sachgerechten Handhabung des Gefahrenbegriffs im umweltbezogenen Gesundheitsrecht ist eine Offenlegung, Typisierung und Standardisierung im Bereich der Dosis-Wirkungs-Abschätzung sehr wichtig. Unter dieser Zielstellung werden hier Methoden zur Ableitung gesundheitlich tolerierbarer Schadstoffzufuhr (TRDWerte) geboten. Für Kanzerogene wird eine besondere Vorgehensweise einge-schlagen. Die abgeleiteten "tolerablen resorbierten Dosen” als Maß für die innere Belastung sind methodisch analog zu TDI-, ADI-Werten zu betrachten. So dient das Handbuch sowohl als Sammlung humantoxikologischer Bewertungsmaßstäbe als auch der ihrer Begründungen.

Aus den vorerst für 19 Stoffe (-gruppen) abgeleiteten TRD-Werten können medienbezogene Werte, wie z.B. Bodenwerte zur Altlastenbewertung, nicht direkt abgelesen werden, weil ergänzend eine nutzungsspezifische Expositionsbetrachtung durchzuführen ist. Ein Vergleich der zu erwartenden Exposition mit der tolerablen Körperbelastung erlaubt dann aber die Abgrenzung noch tolerabler Bodenbelastungen. Da das Werk auf die sogenannte „LAGA-Liste" altlastenrelevanter Stoffe und Stoffgruppen Bezug nimmt, kann wohl damit gerechnet werden, dass mit der Zeit zumindest alle 77 Stoffe (-gruppen) dieser Liste detailliert betrachtet werden.

Für alle Arbeiten, bei denen die Gefahren für den Menschen durch Umweltschadstoffe zu beurteilen sind, werden die hier gebotenen Arbeitsergebnisse sehr bedeutsam. 